\title{
miR-17-5p knockdown inhibits proliferation, autophagy and promotes apoptosis in thyroid cancer via targeting PTEN
}

\author{
Y. P. SHI, G. L. LIU, S. LI, X. L. LIU* \\ Department of Endocrinology, Tangshan Gongren Hospital, Tangshan, China \\ *Correspondence: tsgryylxl2006@126.com
}

Received January 10, 2019 / Accepted June 11, 2019

\begin{abstract}
Thyroid cancer is one common endocrine malignancy with various pathological types. MicroRNAs (miRNAs) play essential roles in development, prognosis and treatment of thyroid cancer. However, the role of miR-17-5p in thyroid cancer progression and its mechanism remain poorly understood. The expressions of miR-17-5p and phosphatase and tensin homolog (PTEN) were measured in thyroid cancer tissues and cells by quantitative real-time polymerase chain reaction or western blot. Cell proliferation and apoptosis were detected by 3-(4,5-dimethyl-2-thiazolyl)-2,5-diphenyl-2$\mathrm{H}$-tetrazolium bromide assay and flow cytometry, respectively. The protein levels of biomarkers in autophagy or protein kinase B (AKT)/mechanistic target of rapamycin (mTOR) pathway were analyzed by western blot. The interaction between miR-17-5p and PTEN was probed by luciferase activity assay. We found that miR-17-5p expression was elevated and PTEN level was reduced in thyroid cancer tissues and cells compared with their corresponding controls. Knockdown of miR-17-5p or overexpression of PTEN suppressed cell proliferation and autophagy but promoted apoptosis in thyroid cancer cells. PTEN was indicated as a target of miR-17-5p and its interference reversed abrogation of miR-17-5p-mediated inhibition of proliferation and autophagy and increase of apoptosis. Moreover, downregulation of miR-17-5p impeded the activation of AKT/mTOR pathway in thyroid cancer cells, which was attenuated by silencing PTEN. Our data supported that knockdown of miR-17-5p upregulated PTEN expression, therefore leading to suppression of the malignancy of thyroid cancer and inactivation of AKT/mTOR pathway, providing a novel avenue for treatment of thyroid cancer.
\end{abstract}

Key words: thyroid cancer, miR-17-5p, PTEN, proliferation, autophagy, apoptosis

Thyroid cancer is the most common endocrine malignancy with approximately $77 \%$ of cases in women worldwide [1]. The thyroid cancer is usually classified as papillary thyroid cancer, follicular thyroid cancer, medullary thyroid cancer and anaplastic thyroid cancer [2]. Among them, papillary thyroid cancer accounts for about $85 \%$ of thyroid cancer cases. Although great developments have been gained in diagnosis and treatment of thyroid cancer, its incidence remains growing [3]. Therefore, there is an urgent need to explore novel strategies for diagnosis and treatment of thyroid cancer.

MicroRNAs (miRNAs) are a class of non-coding RNAs with $\sim 22$ nucleotides, which play essential roles in progression of endocrine-related cancers [4]. The available evidence indicates that miRNAs are associated with thyroid development, function and tumorigenesis [5]. For example, Liu et al. reported that miR-4728 inhibits cell proliferation by regulating mitogen-activated protein kinase (MAPK) pathway in papillary thyroid cancer [6]. Wang et al. revealed that miR-299-5p suppresses migration and invasion by targeting estrogen receptor alpha in papillary thyroid cancer [7]. MiR-17-5p has been suggested to contribute to cell proliferation and tumor growth by regulating p21 in nasopharyngeal carcinoma [8]. Moreover, Cai et al. provided evidence that miR-17-5p promoted cell proliferation and metastasis via regulating transforming growth factor- $\beta$ receptor 2 (TGFBR2) in cervical cancer [9]. In addition, miR-17-5p could induce proliferation and epithelial-mesenchymal transition by inhibiting SRC kinase signaling inhibitor 1 in osteosarcoma [10]. Previous study demonstrated that miR-17-5p is highly expressed in thyroid cancer [11]. However, the effect of miR-17-5p on thyroid cancer progression and its underlying mechanism are largely unknown.

Phosphatase and tensin homolog (PTEN) has been appreciated as an important tumor suppressor and to oppose phosphatidylinositol 3-kinase (PI3K)/protein kinase B (AKT) pathway in human cancers [12]. Accruing works have revealed that PTEN is involved in progression of cancers and 
negatively correlated with AKT signaling in hypopharyngeal carcinoma, non-small cell lung cancer and esophageal squamous cell carcinoma [13-15]. Previous study suggested that loss of PTEN is associated with progression of papillary thyroid cancer [16]. In this study, we sought to investigate the roles of miR-17-5p and PTEN in thyroid cancer progression and explore the potential interaction between miR-17-5p and PTEN/AKT/mechanistic target of rapamycin (mTOR) signaling pathway.

\section{Materials and methods}

Tissue samples. The 25 paired tumor samples and corresponding normal tissues were collected from patients with thyroid cancer from Tangshan Workers Hospital. All samples were snap-frozen and stored at $-80^{\circ} \mathrm{C}$ until used. All participants without history of chemotherapy or radiotherapy have signed the informed consent. This study was approved by the Research Ethics Committee of Tangshan Workers Hospital.

Cell culture and transfection. The human thyroid follicular cell line Nthy-ori 3-1 and thyroid cancer cell lines (TPC-1, FTC-133, B-CPAP and SW579) were purchased from American Tissue Culture Collection (Manassas, VA, USA). All cells were cultured in Dulbecco's Modified Eagle Medium (DMEM; Gibco, Carlsbad, CA, USA) with 10\% fetal bovine serum (FBS; Gibco), $100 \mathrm{U} / \mathrm{ml}$ penicillin and $100 \mu \mathrm{g} / \mathrm{ml}$ streptomycin (Gibco) at $37^{\circ} \mathrm{C}$ and $5 \% \mathrm{CO}_{2}$.

MiR-17-5p antagomir (antagomiR-17-5p), antagomir negative control (antagomiR-NC), miR-17-5p agomir (agomiR-17-5p), agomir negative control (agomiR-NC), PTEN overexpression vector (pc-PTEN), pcDNA empty vector (pc-NC), small interfering RNA (siRNA) against PTEN (si-PTEN) and siRNA negative control (si-NC) were synthesized by Genepharma (Shanghai, China). Cell transfection was performed in TPC-1 and FTC-133 cells by using Lipofectamine 2000 (Invitrogen, Carlsbad, CA, USA) following the manufacturer's instructions.

Quantitative real-time polymerase chain reaction (qRT-PCR). Total RNA was isolated from tissues or cells via using TRIzol reagent (Invitrogen) according to the manufacturer's instructions. The cDNA was obtained by TransScript miRNA First stand cDNA Synthesis SuperMix (TransGen Biotech, Beijing, China) and used for qRT-PCR with SYBR green (Applied Biosystems, Foster City, CA, USA) according to the manufacturer's instructions. The relative expressions of miR-17-5p and PTEN were detected with U6 small RNA or GAPDH as internal control using $2^{-\Delta \Delta C t}$ method [17]. The primers used in this study were listed as follows: miR-17-5p (Forward, 5' - CCAGGATCCTTTATAGTTGTTAGAGTTTG-3'; Reverse, 5'-CGGAATTCTAATCTACTTCACTATCTGCAC-3'), U6 (Forward, 5'-GCTTCGGCAGCACATA-3'; Reverse, 5'-ATGGAACGCTTCACGA-3'), PTEN (Forward, 5'-AAGACAAAGCCAACCGATAC-3'; Reverse, 5'-GAAGTTGAACTGCTAGCCTC-3'), GAPDH (Forward,
5'-ACCTGACCTGCCGTCTAGAA-3'; Reverse, 5'-TCCACCACCCTGTTGCTGTA-3').

Cell proliferation. 3-(4,5-dimethyl-2-thiazolyl)-2,5diphenyl-2-H-tetrazolium bromide (MTT) assay was performed to measure cell proliferation. TPC-1 and FTC-133 cells (5000 cells per well) were seeded into 96-well plates and cultured for 24,48 or $72 \mathrm{~h}$. Then cells were incubated with $0.5 \mathrm{mg} / \mathrm{ml}$ MTT solution (Sigma, St. Louis, MO, USA) for another $4 \mathrm{~h}$. After the solubilization of formazan with dimethyl sulfoxide (DMSO; Thermo Fisher, Wilmington, DE, USA), the absorbance was measured at $570 \mathrm{~nm}$ using a microplate reader (Bio-Rad, Hercules, CA, USA).

Cell apoptosis. Annexin V-fluorescein isothiocyanate (FITC)/propidium iodide (PI) apoptosis detection kit (Solarbio, Beijing, China) was used to analyze cell apoptosis via flow cytometry. After the culture for $72 \mathrm{~h}, \mathrm{TPC}-1$ and FTC-133 cells were washed with cold PBS and resuspended in binding buffer, followed by stained with $5 \mu \mathrm{l}$ Annexin $\mathrm{V}$-FITC for $10 \mathrm{~min}$ and $5 \mu \mathrm{l}$ PI for $5 \mathrm{~min}$ in the dark at room temperature. The positive cells were analyzed by using a flow cytometer (Becton Dickinson, San Jose, CA, USA).

Western blot. After washed with cold PBS, TPC-1 and FTC-133 cells were lysed by using RIPA lysis buffer (Beyotime Biotech, Shanghai, China). Total proteins were quantified by using BCA protein assay kit (Thermo Fisher) and then denatured at $100^{\circ} \mathrm{C}$ for $10 \mathrm{~min}$. Equal amounts of proteins were separated on SDS-PAGE gel and then transferred to polyvinylidene difluoride membranes (Millipore, Billerica, MA, USA). The membranes were blocked with 5\% non-fat milk for $1 \mathrm{~h}$ at room temperature, incubated with primary antibodies overnight at $4^{\circ} \mathrm{C}$ and then interacted with horseradish peroxidase-conjugated secondary antibody for $2 \mathrm{~h}$ at room temperature. The antibodies against LC3, p62, PTEN, AKT, p-AKT (Ser473), mTOR, p-mTOR (Ser2448) or GAPDH and HRP-conjugated secondary antibody were purchased from Cell Signaling Technology (Danvers, MA, USA). GAPDH was used as loading control and the protein signals were visualized using enhanced chemiluminescence chromogenic substrate (Beyotime Biotech).

Luciferase activity assay. The putative binding sites of miR-17-5p and PTEN were predicted by TargetScan online. The 3' untranslated regions (3'-UTR) sequences of PTEN containing wild-type (WT) or mutant (MUT) binding sites of miR-17-5p were cloned into the pMIR-REPORT vectors (Promega, Madison, WI, USA) to synthesize luciferase reporter vectors (WT-PTEN or MUT-PTEN). TPC-1 and FTC-133 cells were co-transfected with luciferase reporter vector, control vector and agomiR-17-5p or agomiR-NC using Lipofectamine 2000 according to the manufacturer's protocols. After the transfection for $48 \mathrm{~h}$, cells were collected for luciferase activity analysis with luciferase assay kit (Promega) according to the manufacturer's instructions.

Statistical analysis. Data were expressed as the mean \pm standard deviation (S.D.) from three independent experiments. The relationship between the expression levels of 
miR-17-5p and PTEN was investigated by spearman rank correlation. The differences between groups were measured by student's $t$ test or one-way analysis of variance (ANOVA) using GraphPad Prism 7 (GraphPad Inc., La Jolla, CA, USA). A p-value $<0.05$ was regarded as statistically significant.

\section{Results}

MiR-17-5p expression is increased in thyroid cancer. To explore the potential role of miR-17-5p in thyroid cancer progression, its expression was first measured in thyroid cancer samples. Results showed that the expression of miR-17-5p was abnormally enhanced by 3.1-fold in thyroid cancer tissues compared with that in adjacent normal tissues (Figure 1A). Moreover, miR-17-5p level was obviously higher in thyroid cancer cells than that in control Nthy-ori 3-1 cells (Figure 1B). Hence, TPC-1 and FTC-133 cells with relative higher miR-17-5p abundance were used for further experiments.

Abrogation of miR-17-5p inhibits progression of thyroid cancer. To investigate the effect of miR-17-5p on thyroid cancer progression in vitro, TPC-1 and FTC-133 cells were transfected with antagomiR-17-5p or antagomiRNC. As a result, the abundance of miR-17-5p was effectively reduced in TPC-1 and FTC-133 cells transfected with antagomiR-17-5p compared with that in antagomiR-NC group (Figure 2A). In addition, knockdown of miR-17-5p significantly decreased proliferation of TPC-1 and FTC-133 cells at $72 \mathrm{~h}$ (Figures 2B and 2C, Figure S1A). Moreover, great increase of apoptosis was displayed in TPC-1 and FTC-133 cells transfected with antagomiR-17-5p compared with that in antagomiR-NC group at $72 \mathrm{~h}$ (Figures 2D and 2E). Besides, inhibition of miR-17-5p led to obvious loss of LC3 II/I ratio and increase of $\mathrm{p} 62$ protein level in TPC-1 and FTC-133 cells (Figures 2F-2H).
PTEN is a target of miR-17-5p. To explore the potential mechanism that allows miR-17-5p participating in thyroid cancer progression, the promising targets of miR-17-5p were predicted by TargetScan. Bioinformatics analysis provided the putative binding sites of miR-17-5p and PTEN, suggesting that PTEN might be a target of miR-17-5p (Figure 3A). To validate this prediction, we constructed WT or MUT luciferase reporter vector. Results showed that overexpression of $\mathrm{miR}-17-5 \mathrm{p}$ resulted in great loss of luciferase activity in TPC-1 and FTC-133 cells transfected with WT-PTEN, while its efficacy was lost in MUT-PTEN group (Figures 3B and 3C). Subsequently, the effect of miR-17-5p on PTEN expression was analyzed in thyroid cancer cells. Addition of miR-17-5p notably decreased the level of PTEN protein in TPC- 1 and FTC-133 cells, while inhibition of miR-17-5p caused an opposite effect (Figures 3D and 3E).

PTEN mRNA level is downregulated in thyroid cancer. Seeing that PTEN was indicated as a target of miR-17-5p, the expression of PTEN was detected in thyroid cancer samples. Compared with normal samples, thyroid cancer tissues showed low expression of PTEN at mRNA level (Figure 4A). Similarly, the mRNA level of PTEN was markedly decreased in TPC-1 and FTC-133 cells compared with that in Nthy-ori 3-1 cells (Figure 4B). Moreover, following the spearman rank correlation analysis, the expression of PTEN mRNA in thyroid cancer tissues was negatively correlated with miR-17-5p abundance $\left(\mathrm{R}^{2}=0.2461, \mathrm{p}=0.0117\right)$ (Figure $\left.4 \mathrm{C}\right)$.

Addition of PTEN represses progression of thyroid cancer. To evaluate the role of PTEN in thyroid cancer progression, TPC-1 and FTC-133 cells were transfected with pc-PTEN or pc-NC. After the transfection, the expression of PTEN protein was effectively elevated in TPC- 1 and FTC-133 cells transfected with pc-PTEN compared with that in pc-NC group (Figures 5A and 5B). Moreover, overexpression of PTEN significantly protected against proliferation of
A

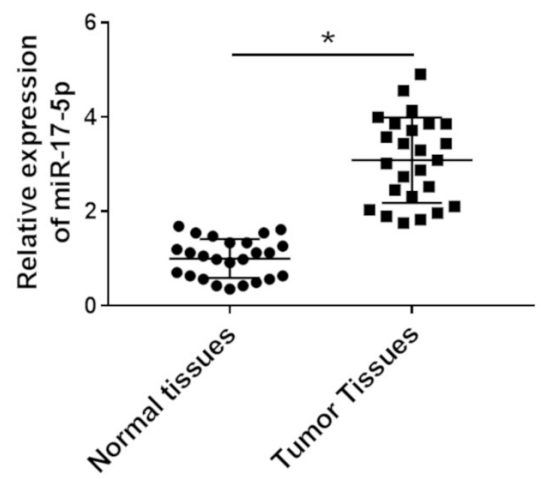

B

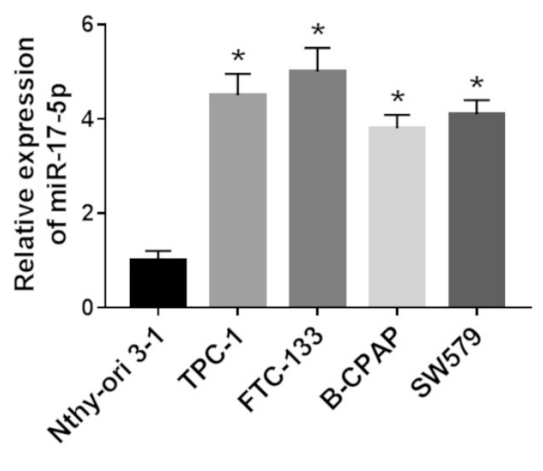

Figure 1. The expression of miR-17-5p is upregulated in thyroid cancer samples. A) The level of miR-17-5p was measured in thyroid cancer tissues and adjacent normal tissues by $q R T-P C R, n=25$. B) The abundance of miR-17-5p was detected in thyroid cancer cells and normal cell line by $q R T-P C R$. ${ }^{*} \mathbf{p}<0.05$. 
A

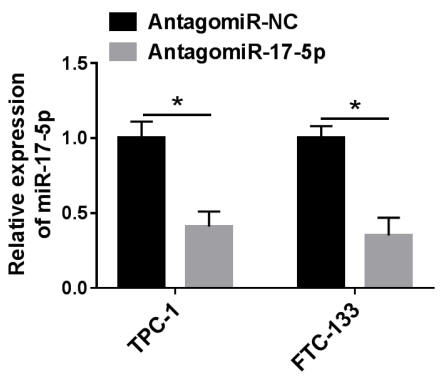

D

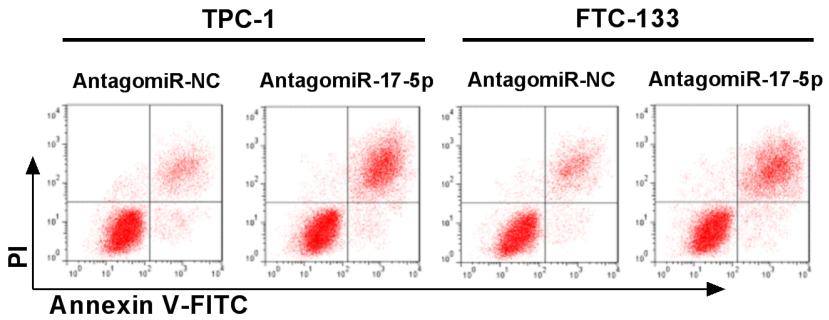

$\mathbf{F}$

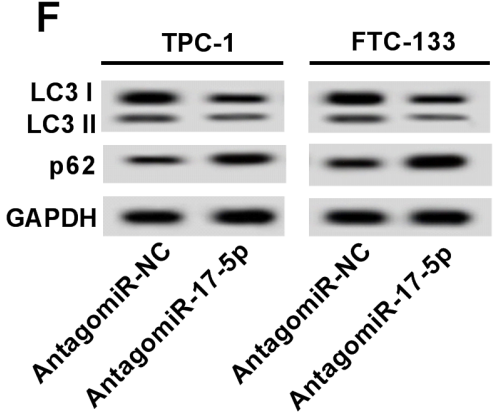

B

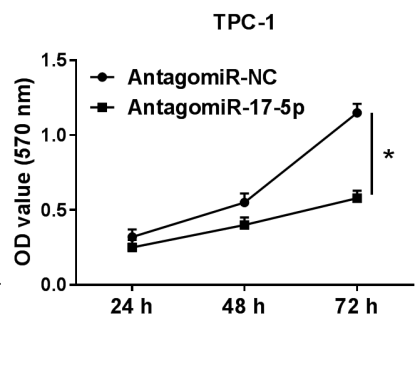

G

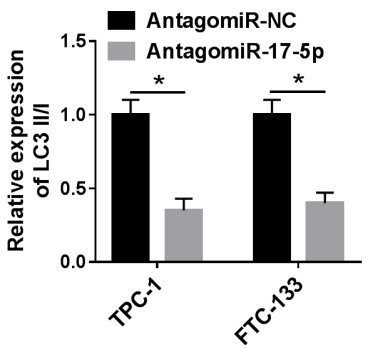

C

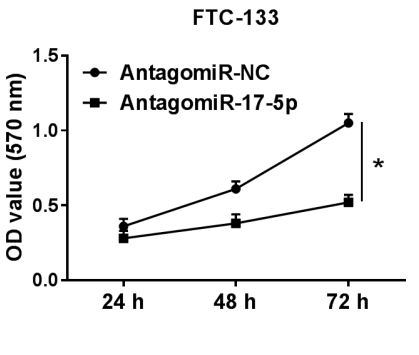

E

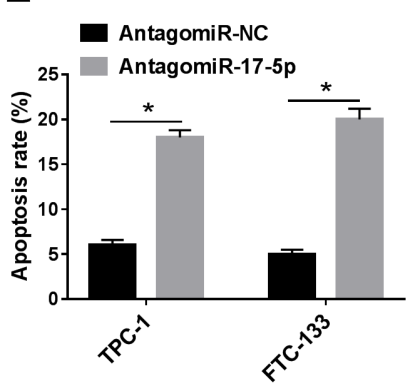

$\mathrm{H}$

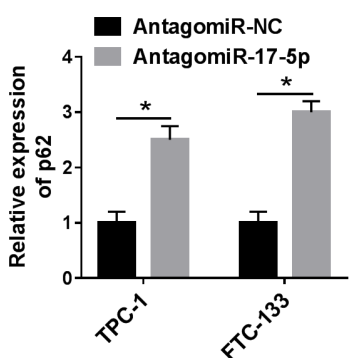

Figure 2. MiR-17-5p knockdown inhibits proliferation and autophagy but promotes apoptosis in thyroid cancer cells. A) The expression of miR-17-5p was measured in TPC-1 and FTC-133 cells transfected with antagomiR-17-5p or antagomiR-NC by qRT-PCR. B and C) Cell proliferation was detected in TPC-1 and FTC-133 cells transfected with antagomiR-17-5p or antagomiR-NC at 24, 48 or $72 \mathrm{~h}$ by MTT assay. D and E) Cell apoptosis was examined in TPC-1 and FTC-133 cells transfected with antagomiR-17-5p or antagomiR-NC by flow cytometry. F-H) The levels of LC3 II/I ratio and p62 protein were analyzed in TPC-1 and FTC-133 cells transfected with antagomiR-17-5p or antagomiR-NC by western blot. ${ }^{\star}$ p $<0.05$.

TPC-1 and FTC-133 cells (Figures 5C and 5D, Figure S1B). However, abundant accumulation of PTEN resulted in great increase of apoptosis in TPC-1 and FTC-133 cells compared with pc-NC group at $72 \mathrm{~h}$ (Figures 5E and 5F). In addition, obvious suppression of LC3 II/I ratio and increase of p62 levels were detected in TPC-1 and FTC-133 cells transfected with pc-PTEN compared with that in pc-NC group (Figures 5G-5I).

Silencing PTEN reverses downregulation of miR-175 p-mediated inhibition of thyroid cancer progression. To explore whether PTEN was required for miR-17-5pmediated progression of thyroid cancer, TPC-1 and FTC-133 cells were transfected with antagomiR-NC, antagomiR-17-5p, antagomiR-17-5p and si-NC or si-PTEN. As a result, transfection efficacy analysis revealed that transfection of si-PTEN attenuated the promoted effect of miR-17-5p knockdown on PTEN protein level in TPC- 1 and FTC-133 cells (Figures 6A and 6B). MTT assay showed that interference of PTEN attenuated knockdown of miR-17-5p-mediated inhibition of proliferation in TPC-1 and FTC-133 cells (Figures 6C and 6D, Figure S1C). Moreover, absence of PTEN abated downregulation of miR-17-5p-induced apoptosis of TPC-1 and FTC-133 cells (Figure 6E). Additionally, depletion of PTEN evidently alleviated the regulatory effect of miR-17-5p exhaustion on LC3 II/I ratio and p62 expression in TPC-1 and FTC-133 cells (Figures 6F-6H).

MiR-17-5p knockdown inhibits AKT/mTOR pathway by regulating PTEN in thyroid cancer cells. To further elucidate the molecular mechanism, the potential signaling pathway was explored in TPC-1 and FTC-133 cells trans- 
A

WT PTEN 3'UTR 5'-GgAUUAAUAAAGAUG GCACUUUC-3'

miR-17-5p 3'-GAUGGACGUGACAUUCGUGAAAC-5'

MUT PTEN 3'UTR 5'-GGAUUAAUAAAGAUGCGUGAAAC-3'

B

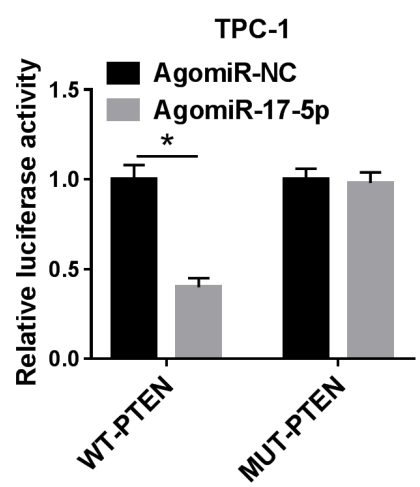

D

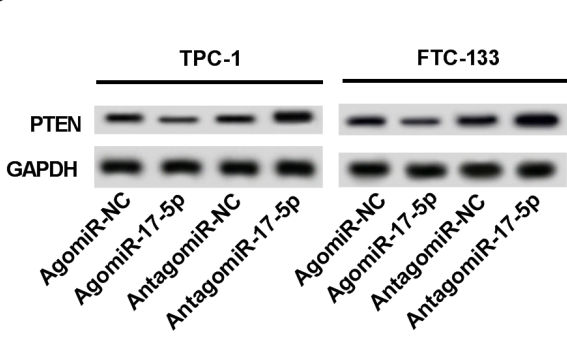

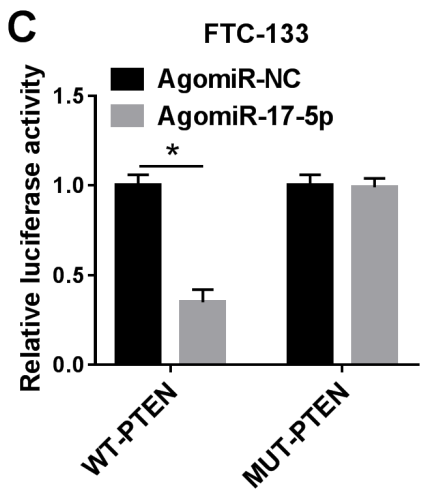

E

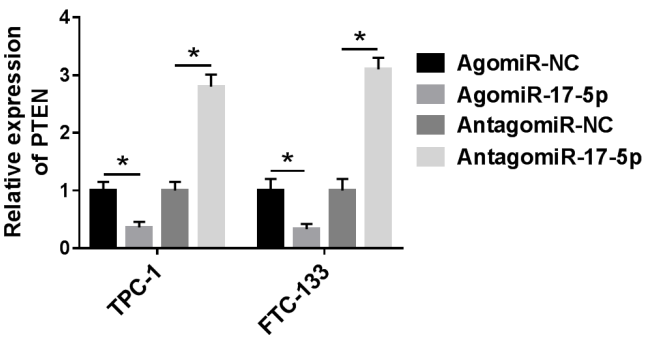

Figure 3. PTEN is a target of miR-17-5p. A) The potential binding sites of miR-17-5p and PTEN were provided by TargetScan. B and C) Luciferase activity was analyzed in TPC-1 and FTC-133 cells co-transfected with WT-PTEN or MUT-PTEN and agomiR-17-5p or agomiR-NC. D and E) The expression of PTEN protein was measured in TPC-1 and FTC-133 cells transfected with agomiR-NC, agomiR-17-5p, antagomiR-17-5p or antagomiR-NC by western blot. ${ }^{\star} \mathrm{p}<0.05$.

A

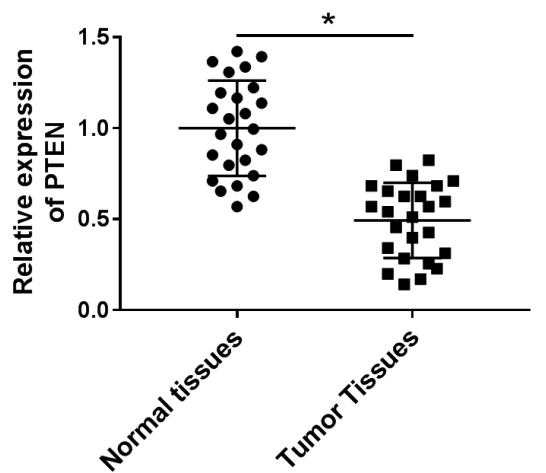

B

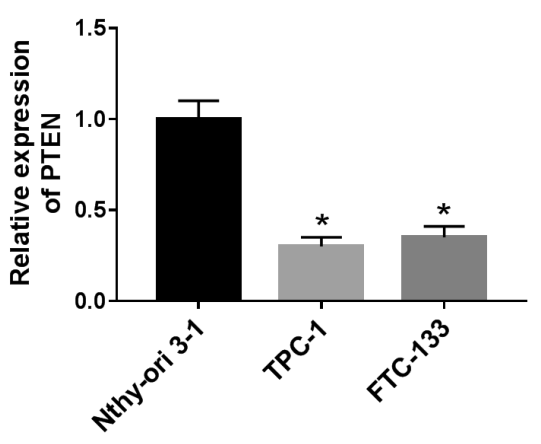

C

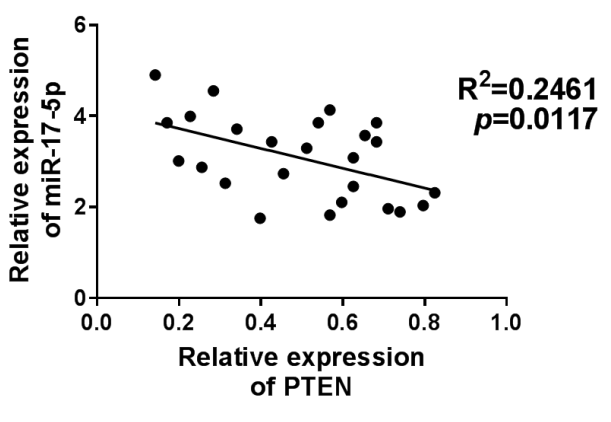

Figure 4. The expression of PTEN is downregulated in thyroid cancer samples. A and B) The expression of miR-17-5p was measured in thyroid cancer tissues $(n=25)$ and cells by qRT-PCR. C) The relationship between expressions of miR-17-5p and PTEN in thyroid cancer tissues was explored by spearman rank correlation. ${ }^{*} \mathbf{p}<0.05$. 
A

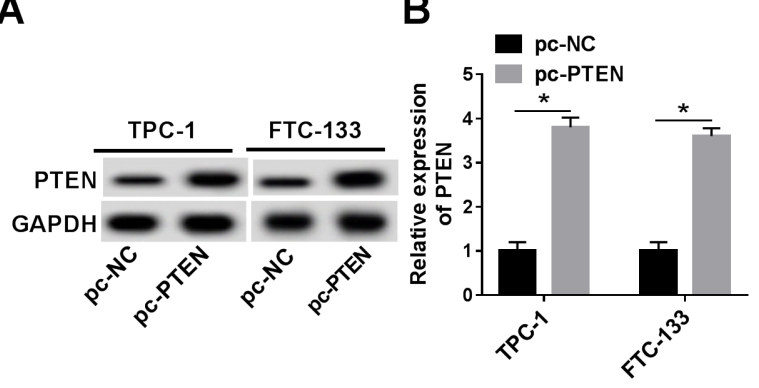

C

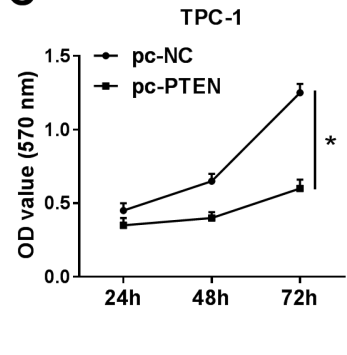

D

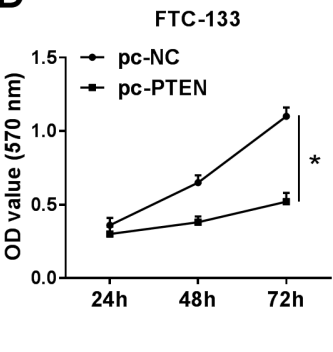

E

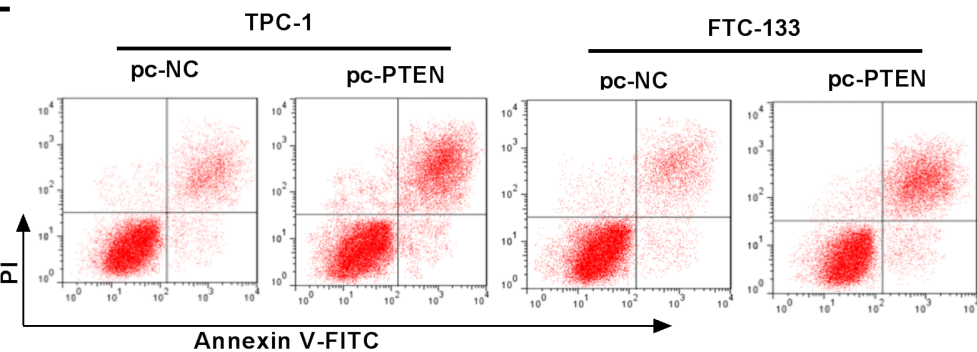

$\mathbf{m} \quad \mathbf{m o c}_{\mathrm{pc}-\mathrm{NC}}$

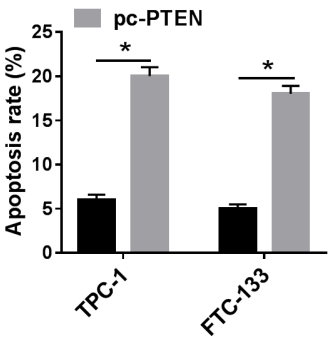

G
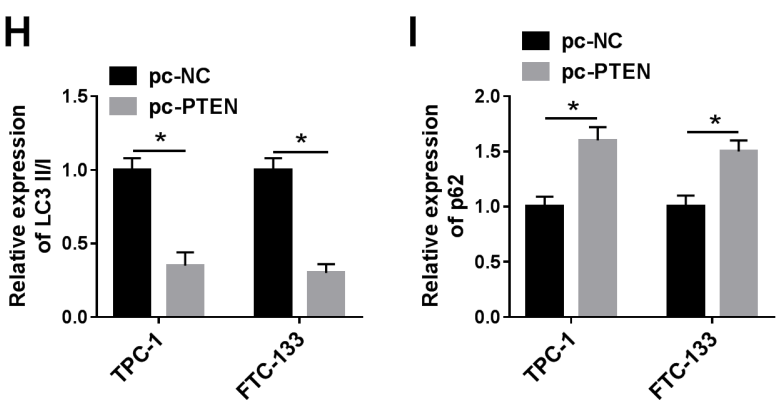

Figure 5. PTEN overexpression suppresses proliferation and autophagy but increases apoptosis in thyroid cancer cells. A and B) The expression of PTEN protein was measured in TPC-1 and FTC-133 cells transfected with pc-PTEN or pc-NC by western blot. Cell proliferation (C and D), apoptosis (E and F) and autophagy (G-I) were detected in TPC-1 and FTC-133 cells transfected with pc-PTEN or pc-NC by MTT assay, flow cytometry or western blot, respectively. ${ }^{\star} \mathbf{p}<0.05$.

fected with antagomiR-NC, antagomiR-17-5p, antagomiR17-5p and si-NC or si-PTEN. Results showed that abrogation of miR-17-5p obviously limited the phosphorylation of AKT (Ser473), which was overturned by interference of PTEN, while it showed little effect on total AKT level in TPC-1 and FTC-133 cells (Figures 7A-C). Similarly, deficiency of miR-17-5p significantly hindered the activation of mTOR (Ser2448), which was weakened by silencing of PTEN, whereas it failed to show efficacy for total mTOR level in TPC- 1 and FTC-133 cells (Figures 7D and 7E).

\section{Discussion}

MiRNA supplementary therapy has become one promising strategy for the treatment of different types of thyroid cancer [18]. MiR-17-5p, as an attractive miRNA, has been reported to serve as an important oncogene in human cancers, such as osteosarcoma, cervical cancer, nasopharyngeal, breast cancer, pancreatic cancer and gastric cancer [8-10, 19-21]. However, its role in thyroid cancer remains largely unclear. In this study, we first investigated the effect of miR-17-5p on cell proliferation, apoptosis and autophagy in thyroid cancer and then explored the potential underlying mechanism.

Here, we first measured the abundance of miR-17-5p in thyroid cancer tissues and cells and found that miR-17-5p expression was enhanced in cancer tissues and cells compared with that in controls, which is also in agreement with former report [11]. Subsequently, loss-of-function experiments revealed that knockdown of miR-17-5p inhibited cell proliferation but increased apoptosis in thyroid cancer cells, suggesting miR-17-5p as an oncogene in thyroid cancer. Moreover, autophagy has been reported to be involved in initiation, progression and therapeutics of thyroid cancer [22]. Hence, this study also evaluated the effect of miR-17-5p on expressions of autophagy-related protein (LC3 and p62). The reduction of ratio of LC3 II/I and increase of p62 
A

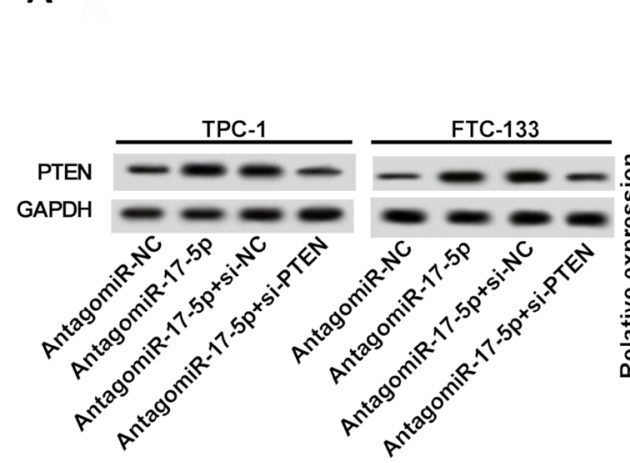

B

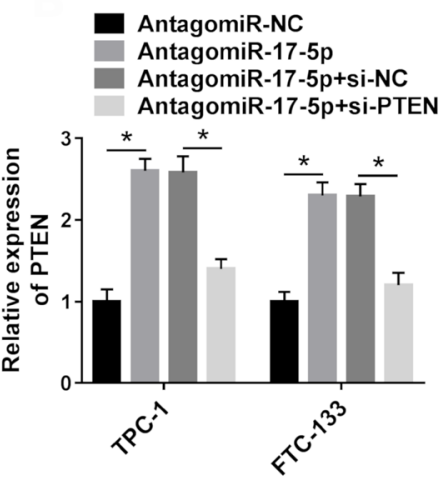

C

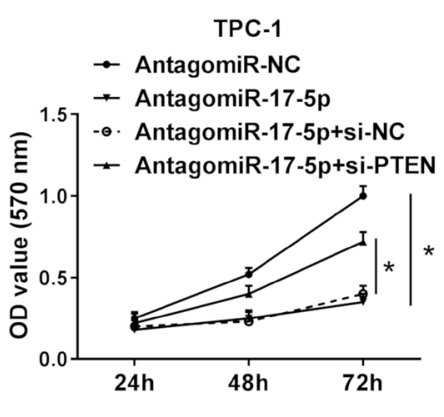

D

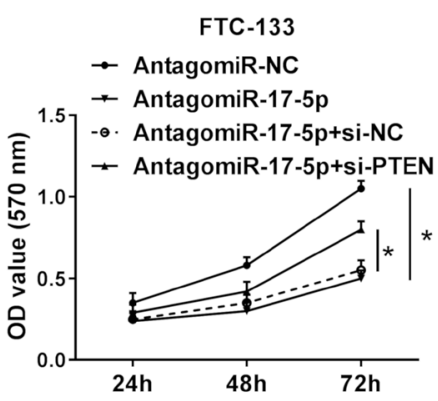

$\mathbf{F}$

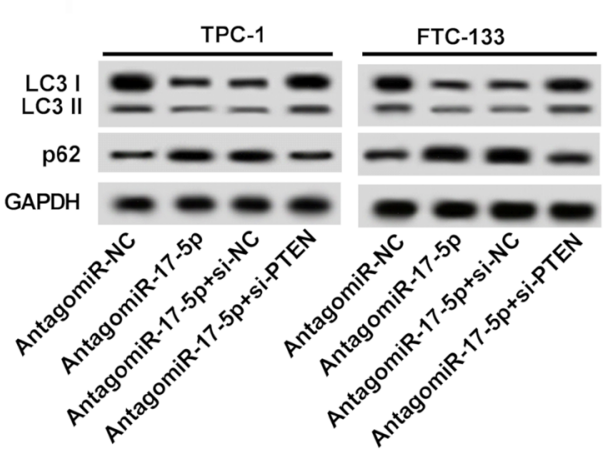

E

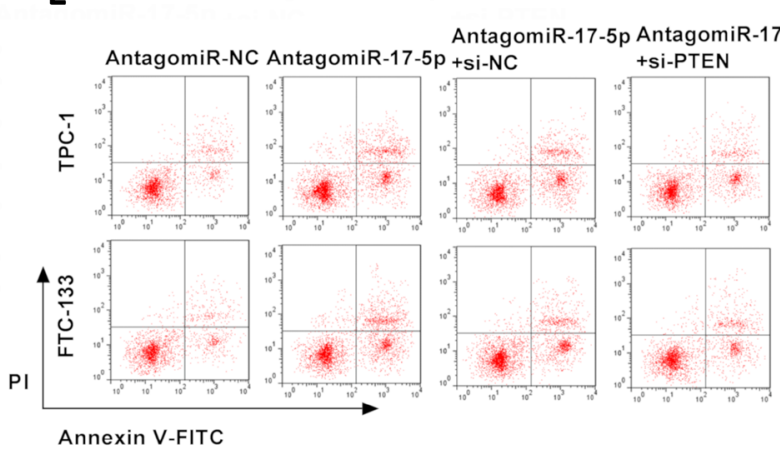

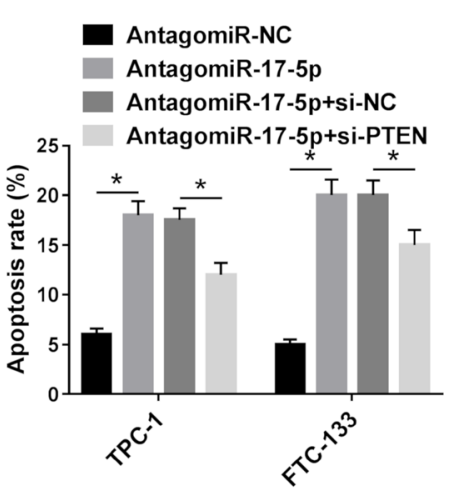

\section{H}

AntagomiR-NC
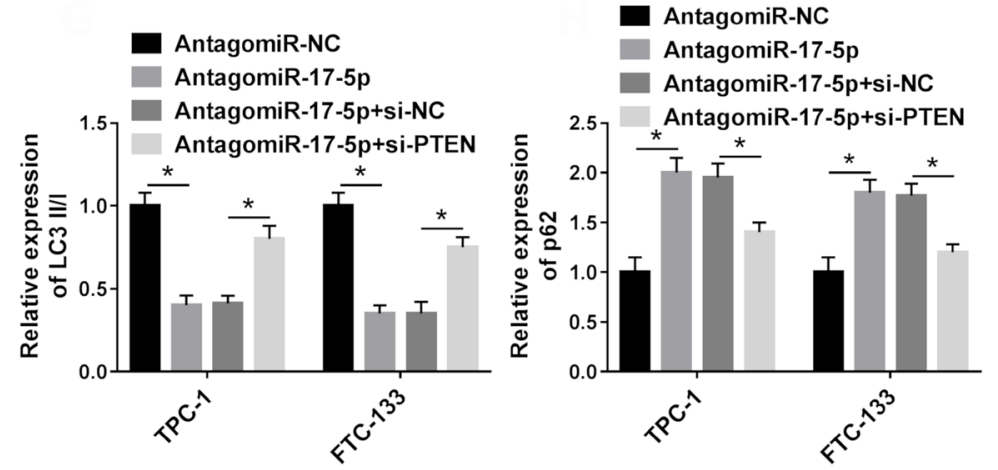

Figure 6. Interference of PTEN reverses exhaustion of miR-17-5p-mediated progression of thyroid cancer. The PTEN protein levels (A and B), cell proliferation (C and D), apoptosis (E) and autophagy (F-H) were measured in TPC-1 and FTC-133 cells transfected with antagomiR-NC, antagomiR17-5p, antagomiR-17-5p and si-NC or si-PTEN by MTT assay, flow cytometry or western blot, respectively. ${ }^{\star}$ p $<0.05$.

expression uncovered that abrogation of miR-17-5p blocked autophagy formation in thyroid cancer cells. Functional miRNAs are known to regulate target expressions by binding their 3'-UTR sequences. Therefore, to validate the underlying mechanism that allows miR-17-5p participating in above progress, we analyzed the potential targets of miR-17-5p. A number of investigators have reported the potential relationship of miR-17-5p and PTEN in gastric cancer, breast cancer, pancreatic cancer and hepatocellular cancer [23-26]. Nevertheless, there is no direct evidence in support of the inter- action between miR-17-5p and PTEN in thyroid cancer. In the present work, luciferase reporter assay showed that introduction of miR-17-5p reduced the luciferase activity of WT-PTEN luciferase reporter vector in thyroid cancer cells, indicating that PTEN might also be a functional target of miR-17-5p in thyroid cancer.

PTEN has been indicated as an important tumor suppressor and restoration of its activity created novel avenue for cancer prevention and treatment [27]. Han et al. have reported that inactivation of PTEN/PI3K/AKT pathway 
A

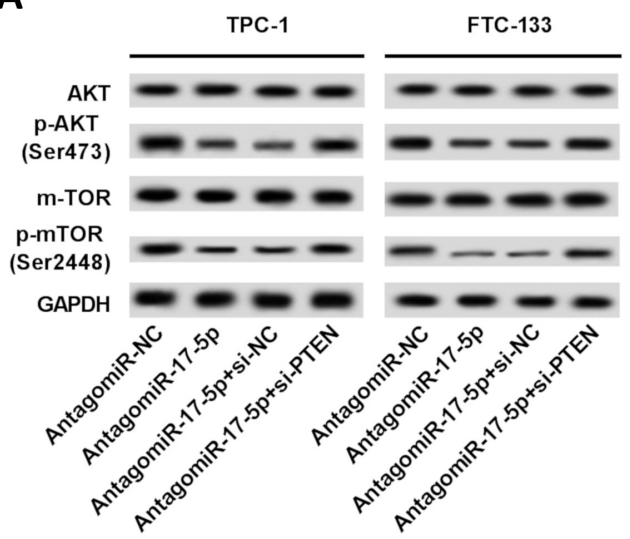

B

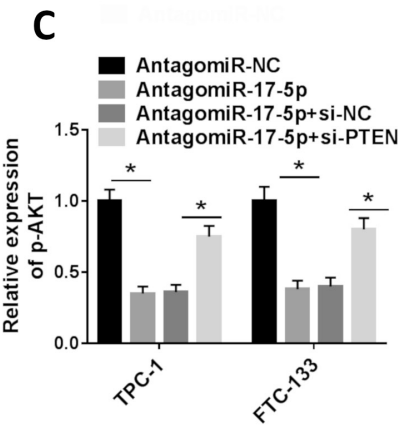

D

E
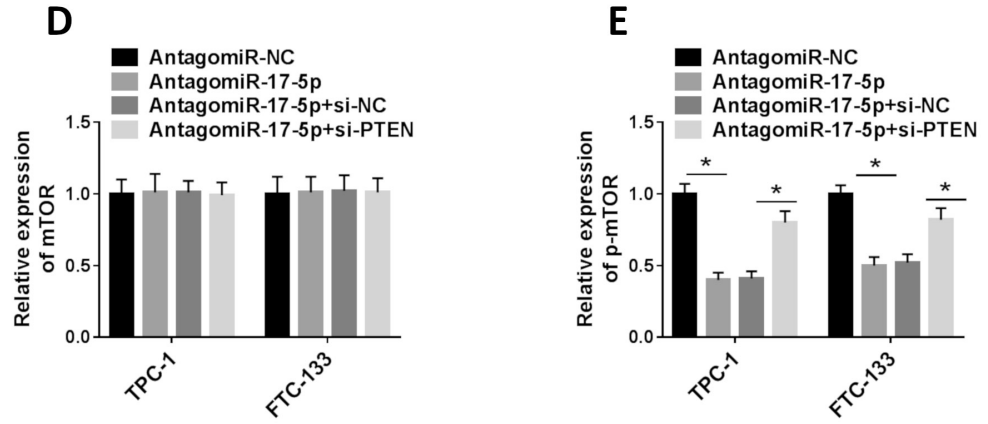

Figure 7. MiR-17-5p regulates AKT/mTOR pathway in thyroid cancer cells. A-E) The protein expressions of p-AKT (Ser473), AKT, p-mTOR (Ser2448) and mTOR were measured in TPC-1 and FTC-133 cells transfected with antagomiR-NC, antagomiR-17-5p, antagomiR-17-5p and si-NC or si-PTEN by western blot. ${ }^{*} \mathrm{p}<0.05$.

suppressed tumorigenesis of papillary thyroid cancer [28]. Moreover, restoration of PTEN was suggested to attenuate miR-146b-induced migration and invasion in thyroid cancer [29]. These works indicated that PTEN might function as a tumor suppressor in thyroid cancer. Similar to our effort, qRT-PCR assay showed that PTEN abundance was decreased in thyroid cancer tissues and cells and its addition suppressed proliferation and autophagy but promoted apoptosis in thyroid cancer cells, which also indicated PTEN as thyroid cancer suppressor. In addition, rescue experiments revealed that interference of PTEN weakened the regulatory effect of knockdown of miR-17-5p on proliferation, apoptosis and autophagy. This reflected that miR-17-5p addressed progression of thyroid cancer by targeting PTEN.
AKT/mTOR signaling pathway is positively implicated in development and progression of thyroid cancer $[30,31]$. The available evidences have indicated PTEN as critical mediator in $\mathrm{PI} 3 \mathrm{~K} / \mathrm{AKT} / \mathrm{mTOR}$ pathway in human cancers, including thyroid cancer [32,33]. Thus, to further underlie the potential mechanism, we speculated whether miR-17-5p might facilitate progression of thyroid cancer by regulating PTEN/AKT/ mTOR pathway. Western blot analysis revealed that inhibition of miR-17-5p impeded the phosphorylation of AKT and mTOR, which was reversed by silencing PTEN. These data validated that miR-17-5p knockdown could inactivate AKT/ mTOR pathway by PTEN upregulation. In addition, mTOR signaling is suggested as an inhibitor of autophagy-associated cell death [34]. However, our study showed that knockdown 
of miR-17-5p-mediated proliferation inhibition was induced by apoptosis but not autophagy. This stimulated us to hypothesize that the autophagy might be modulated by mTOR independent pathway, which needs further investigation.

In conclusion, miR-17-5p expression was increased in thyroid cancer tissues and cells in comparison to that in corresponding controls. MiR-17-5p deficiency inhibited cell proliferation and autophagy, induced apoptosis, and blocked the activation of AKT/mTOR pathway in thyroid cancer cells, possibly by targeting PTEN. This may elucidate a new regulatory mechanism for understanding the progression of thyroid cancer and provide a novel avenue for treatment of thyroid cancer.

Supplementary information is available in the online version of the paper.

\section{References}

[1] KITAHARA CM, SOSA JA. The changing incidence of thyroid cancer. Nat Rev Endocrinol 2016; 12: 646-653. https:// doi.org/10.1038/nrendo.2016.110

[2] BALOCH ZW, LIVOLSI VA. Special types of thyroid carcinoma. Histopathology 2018; 72: 40-52. https://doi. org/10.1111/his.13348

[3] SCHNEIDER DF, CHEN H. New developments in the diagnosis and treatment of thyroid cancer. CA Cancer J Clin 2013; 63: 374-394. https://doi.org/10.3322/caac.21195

[4] KLINGE CM. Non-coding RNAs: long non-coding RNAs and microRNAs in endocrine-related cancers. Endocr Relat Cancer 2018; 25: R259-R282. https://doi.org/10.1530/ERC17-0548

[5] FUZIWARA CS, KIMURA ET. MicroRNAs in thyroid development, function and tumorigenesis. Mol Cell Endocrinol 2017; 456: 44-50. https://doi.org/10.1016/j.mce.2016.12.017

[6] LIU Z, ZHANG J, GAO J, LI Y. MicroRNA-4728 mediated regulation of MAPK oncogenic signaling in papillary thyroid carcinoma. Saudi J Biol Sci 2018; 25: 986-990. https://doi. org/10.1016/j.sjbs.2018.05.014

[7] WANG Z, HE L, SUN W, QIN Y, DONG W et al. miRNA299-5p regulates estrogen receptor alpha and inhibits migration and invasion of papillary thyroid cancer cell. Cancer Manag Res 2018; 10: 6181-6193. https://doi.org/10.2147/ CMAR.S182625

[8] CHEN C, LU Z, YANG J, HAO W, QIN Y et al. MiR-17-5p promotes cancer cell proliferation and tumorigenesis in nasopharyngeal carcinoma by targeting p21. Cancer Med 2016; 5: 3489-3499. https://doi.org/10.1002/cam4.863

[9] CAI N, HU L, XIE Y, GAO JH, ZHAI W et al. MiR-17-5p promotes cervical cancer cell proliferation and metastasis by targeting transforming growth factor-beta receptor 2. Eur Rev Med Pharmacol Sci 2018; 22: 1899-1906. https://doi. org/10.26355/eurrev_201804_14712

[10] ZHAO X, XU Y, SUN X, MA Y, ZHANG Y et al. miR-17-5p promotes proliferation and epithelial-mesenchymal transition in human osteosarcoma cells by targeting SRC kinase signaling inhibitor 1. J Cell Biochem 2019; 120: 5495-5504. https://doi.org/10.1002/jcb.27832
[11] TAKAKURA S, MITSUTAKE N, NAKASHIMA M, NAMBA $\mathrm{H}$, SAENKO VA et al. Oncogenic role of miR-17-92 cluster in anaplastic thyroid cancer cells. Cancer Sci 2008; 99: 11471154. https://doi.org/10.1111/j.1349-7006.2008.00800.x

[12] WORBY CA, DIXON JE. PTEN. Annu Rev Biochem 2014; 83: 641-669. https://doi.org/10.1146/annurev-biochem-082411-113907

[13] WANG Q, TAN L, LIU J, ZHAO J, ZHOU X et al. MicroRNA98/PTEN/AKT pathway inhibits cell proliferation and malignant progression of hypopharyngeal carcinoma by MTDH. Oncol Rep 2019; 41: 863-874. https://doi. org/10.3892/or.2018.6904

[14] XU F, GAO X, PAN H. Pectolinarigenin inhibits nonsmall cell lung cancer progression by regulating the PTEN/PI3K/ AKT signaling pathway. Oncol Rep 2018; 40: 3458-3468. https://doi.org/10.3892/or.2018.6759

[15] HE Y, MINGYAN E, WANG C, LIU G, SHI M et al. CircVRK1 regulates tumor progression and radioresistance in esophageal squamous cell carcinoma by regulating miR624-3p/PTEN/PI3K/AKT signaling pathway. Int J Biol Macromol 2019; 125: 116-123. https://doi.org/10.1016/j. ijbiomac.2018.11.273

[16] BEG S, SIRAJ AK, JEHAN Z, PRABAKARAN S, AL-SOBHI SS et al. PTEN loss is associated with follicular variant of Middle Eastern papillary thyroid carcinoma. Br J Cancer 2015; 112: 1938-1943. https://doi.org/10.1038/bjc.2015.169

[17] LIVAK KJ, SCHMITTGEN TD. Analysis of Relative Gene Expression Data Using Real-Time Quantitative PCR and the $2-\Delta \Delta$ C T Method. Methods 2001; 25: 402-408. https://doi. org/10.1006/meth.2001.1262

[18] PISHKARI S, PARYAN M, HASHEMI M, BALDINI E, MOHAMMADI-YEGANEH S. The role of microRNAs in different types of thyroid carcinoma: a comprehensive analysis to find new miRNA supplementary therapies. J Endocrinol Invest 2018; 41: 269-283. https://doi.org/10.1007/s40618017-0735-6

[19] WANG Y, LI J, DAI L, ZHENG J, YI Z et al. MiR-17-5p may serve as a novel predictor for breast cancer recurrence. Cancer Biomark 2018; 22: 721-726. https://doi.org/10.3233/ CBM-181228

[20] ZHU Y, GU J, LI Y, PENG C, SHI M et al. MiR-17-5p enhances pancreatic cancer proliferation by altering cell cycle profiles via disruption of RBL2/E2F4-repressing complexes. Cancer Lett 2018; 412: 59-68. https://doi.org/10.1016/j.canlet.2017.09.044

[21] CHEN P, ZHAO H, HUANG J, YAN X, ZHANG Y et al. MicroRNA-17-5p promotes gastric cancer proliferation, migration and invasion by directly targeting early growth response 2. Am J Cancer Res 2016; 6: 2010-2020.

[22] NETEA-MAIER RT, KLUCK V, PLANTINGA TS, SMIT JW. Autophagy in thyroid cancer: present knowledge and future perspectives. Front Endocrinol (Lausanne) 2015; 6: 22. https://doi.org/10.3389/fendo.2015.00022

[23] LU R, ZHAO G, YANG Y, JIANG Z, CAI J et al. Long noncoding RNA HOTAIRM1 inhibits cell progression by regulating miR-17-5p/ PTEN axis in gastric cancer. J Cell Biochem 2019; 120: 4952-4965. https://doi.org/10.1002/jcb.27770 
[24] WU SY, WU AT, YUAN KS, LIU SH. Brown Seaweed Fucoidan Inhibits Cancer Progression by Dual Regulation of mir-29c/ADAM12 and miR-17-5p/PTEN Axes in Human Breast Cancer Cells. J Cancer 2016; 7: 2408-2419. https:// doi.org/10.7150/jca.15703

[25] GU J, WANG D, ZHANG J, ZHU Y, LI Y et al. GFRalpha2 prompts cell growth and chemoresistance through downregulating tumor suppressor gene PTEN via Mir-17-5p in pancreatic cancer. Cancer Lett 2016; 380: 434-441. https:// doi.org/10.1016/j.canlet.2016.06.016

[26] SHAN SW, FANG L, SHATSEVA T, RUTNAM ZJ, YANG X et al. Mature miR-17-5p and passenger miR-17-3p induce hepatocellular carcinoma by targeting PTEN, GalNT7 and vimentin in different signal pathways. J Cell Sci 2013; 126: 1517-1530. https://doi.org/10.1242/jcs. 122895

[27] LEE YR, CHEN M, PANDOLFI PP. The functions and regulation of the PTEN tumour suppressor: new modes and prospects. Nat Rev Mol Cell Biol 2018; 19: 547-562. https://doi. org/10.1038/s41580-018-0015-0

[28] HAN M, CHEN L, WANG Y. miR-218 overexpression suppresses tumorigenesis of papillary thyroid cancer via inactivation of PTEN/PI3K/AKT pathway by targeting Runx2. Onco Targets Ther 2018; 11: 6305-6316. https://doi. org/10.2147/OTT.S172152
[29] RAMIREZ-MOYA J, WERT-LAMAS L, SANTISTEBAN P. MicroRNA-146b promotes PI3K/AKT pathway hyperactivation and thyroid cancer progression by targeting PTEN. Oncogene 2018; 37: 3369-3383. https://doi.org/10.1038/ s41388-017-0088-9

[30] MENG X, DONG Y, YU X, WANG D, WANG S et al. MREG suppresses thyroid cancer cell invasion and proliferation by inhibiting Akt-mTOR signaling. Biochem Biophys Res Commun 2017; 491: 72-78. https://doi.org/10.1016/j. bbrc.2017.07.044

[31] YI H, YE X, LONG B, YE T, ZHANG L et al. Inhibition of the AKT/mTOR Pathway Augments the Anticancer Effects of Sorafenib in Thyroid Cancer. Cancer Biother Radiopharm 2017; 32: 176-183. https://doi.org/10.1089/cbr.2017.2187

[32] SANSAL I, SELLERS WR. The biology and clinical relevance of the PTEN tumor suppressor pathway. J Clin Oncol 2004; 22: 2954-2963. https://doi.org/10.1200/JCO.2004.02.141

[33] NOZHAT Z, HEDAYATI M. PI3K/AKT Pathway and Its Mediators in Thyroid Carcinomas. Mol Diagn Ther 2016; 20 : 13-26. https://doi.org/10.1007/s40291-015-0175-y

[34] LI Y, ZHANG L, LI K, LI J, XIANG R et al. ZNF32 inhibits autophagy through the mTOR pathway and protects MCF-7 cells from stimulus-induced cell death. Sci Rep 2015; 5: 9288. https://doi.org/10.1038/srep09288 


\section{miR-17-5p knockdown inhibits proliferation, autophagy and promotes apoptosis in thyroid cancer via targeting PTEN}

Y. P. SHI, G. L. LIU, S. LI, X. L. LIU*

Supplementary Information
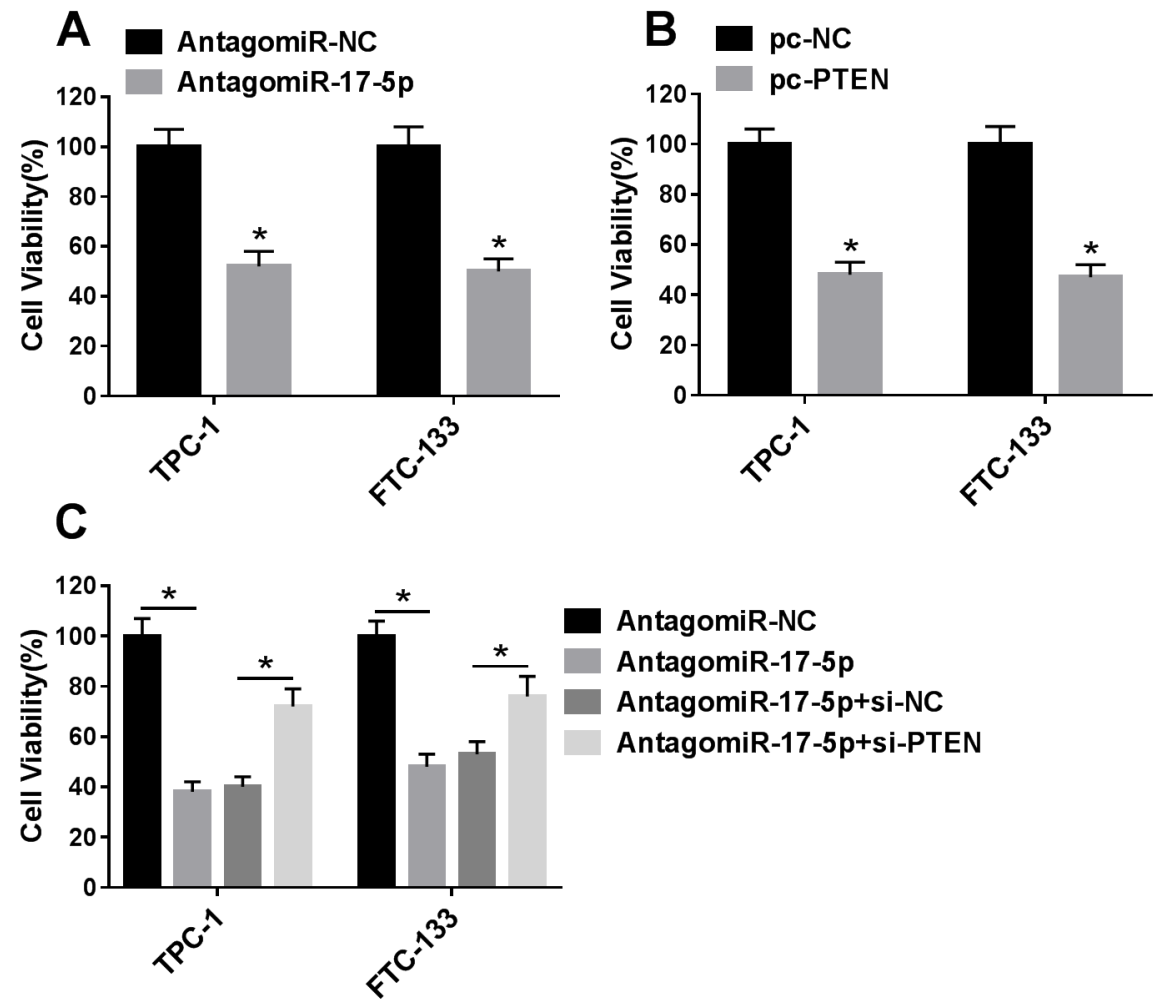

AntagomiR-NC

AntagomiR-17-5p

AntagomiR-17-5p+si-NC

AntagomiR-17-5p+si-PTEN

Figure S1. The cell viability of TPC-1 and FTC-133. A) Cell viability was measured in TPC-1 and FTC-133 cells transfected with antagomiR-NC or antagomiR-17-5p. B) Cell viability was measured in TPC-1 and FTC-133 cells transfected with pc-NC or pc-PTEN. C) Cell viability was measured in TPC-1 and FTC-133 cells transfected with antagomiR-NC, antagomiR-17-5p, antagomiR-17-5p and si-NC or si-PTEN. ${ }^{*} \mathbf{p}<0.05$. 